\title{
Discovery of narrow X-ray absorption features from the low-mass X-ray binary X 1254-690 with XMM-Newton
}

\author{
L. Boirin and A. N. Parmar
}

\author{
Astrophysics Division, Research and Scientific Support Department of ESA, ESTEC, Postbus 299, \\ 2200 AG Noordwijk, The Netherlands
}

Received 22 April 2003 / Accepted 19 June 2003

\begin{abstract}
We report on two XMM-Newton observations of the low-mass X-ray binary X 1254-690. During an XMM-Newton observation of the low-mass X-ray binary in 2001 January a deep X-ray dip was seen while in a second observation one year later no dips were evident. The $0.5-10 \mathrm{keV}$ EPIC spectra from both non-dipping intervals are very similar being modeled by a disk-blackbody and a power-law continuum with additional structure around $1 \mathrm{keV}$ and narrow absorption features at $7.0 \mathrm{keV}$ and $8.2 \mathrm{keV}$ which are identified with the $\mathrm{K} \alpha$ and $\mathrm{K} \beta$ absorption lines of Fe XXVI. The low-energy structure may be modeled as a $175 \mathrm{eV}(\sigma)$ wide emission line at $\sim 0.95 \mathrm{keV}$. This feature is probably the same structure that was modeled as an absorption edge in an earlier BeppoSAX observation. The absorption line properties show no obvious dependence on orbital phase and are similar in both observations suggesting that the occurrence of such features is not directly related to the presence of dipping activity. Narrow Fe absorption features have been observed from the two superluminal jet sources GRO J1655-40 and GRS 1915+105, and the four low-mass X-ray binaries GX 13+1, MXB 1658-298, X 1624-490 and X 1254-690. Since the latter 3 sources are dipping sources, which are systems viewed close to the accretion disk plane, and the two microquasars are thought to be viewed at an inclination of $\sim 70^{\circ}$, this suggests that these features are more prominent when viewed at high-inclination angles. This, together with the lack of any orbital dependence, implies a cylindrical geometry for the absorbing material.
\end{abstract}

Key words. accretion, accretion disks - stars: individual: X 1254-690 - stars: neutron - X-rays: general

\section{Introduction}

During EXOSAT observations in 1984 and 1985 the low-mass X-ray binary (LMXRB) source X 1254-690 exhibited irregular dips in X-ray intensity that repeated every $3.88 \pm 0.15 \mathrm{hr}$ (Courvoisier et al. 1986). The duration of each dip was $\sim 0.8 \mathrm{hr}$ with a mean reduction in 1-10 keV intensity of $\sim 95 \%$. From optical $V$-band observations of the 19th magnitude companion Motch et al. (1987) determined an optical period of $3.9334 \pm$ $0.0002 \mathrm{hr}$, consistent with the mean X-ray dip recurrence interval. The dips are due to obscuration in the thickened outer regions of an azimuthally structured accretion disk (White \& Swank 1982). The dips were still present during Ginga observations in 1990 (Uno et al. 1997), but were not detected by RXTE in 1997 (Smale \& Wachter 1999). Simultaneous optical observations revealed that the mean optical magnitude was unchanged, but that the amplitude of the optical variability had declined (Smale \& Wachter 1999). The dips were also absent during a BeppoSAX observation in 1998 (Iaria et al. 2001). A likely explanation for the absence of dips is that the vertical structure in the outer region of the accretion disk had decreased in size so that the central X-ray source could be viewed directly (Smale \& Wachter 1999). The dips had re-appeared by

Send offprint requests to: L. Boirin,

e-mail: lboirin@rssd.esa.int the time of a RXTE observation in 2001 May, but were again not present in 2001 December (Smale et al. 2002). The 0.1$100 \mathrm{keV}$ BeppoSAX spectrum of X 1254-690 may be modeled by the combination of a multicolor disk-blackbody with an inner temperature of $\sim 0.85 \mathrm{keV}$ and a Comptonized component with an electron temperature of $\sim 2 \mathrm{keV}$ and an optical depth of $\sim 19$. A hard excess is visible around $20 \mathrm{keV}$ which may be accounted for using a bremsstrahlung model with a temperature of $\sim 20 \mathrm{keV}$ (Iaria et al. 2001). There was also evidence in the BeppoSAX observation for an absorption edge at $\sim 1.27 \mathrm{keV}$ with an optical depth of $\sim 0.15$. Iaria et al. (2001) propose that the Comptonized component could originate in a spherical cloud, or boundary layer, surrounding the neutron star while the bremsstrahlung component probably originates in an extended accretion disk corona with a radius of $10^{10} \mathrm{~cm}$ and a mean electron density of $\sim 1.7 \times 10^{14} \mathrm{~cm}^{-3}$.

The PN camera (Strüder et al. 2001) of the European Photon Imaging Camera (EPIC) on-board XMM-Newton (Jansen et al. 2001), with its good high-energy sensitivity and spectral resolution, is proving to be very successful in discovering narrow absorption features from highly ionized $\mathrm{Fe}$ and other metals in the spectra of LBXRBs.

Narrow X-ray absorption lines were first detected from the superluminal jet sources GROJ1655-40 (Ueda et al. 1998) and GRS 1915+105 (Kotani et al. 2000), and it has 
been suggested that these features are related to the jet formation mechanism. With the detection of X-ray absorption lines in GX 13+1, MXB 1658-298, X 1624-490 and now X 1254-690, this now appears unlikely. X-ray absorption lines were seen from GX 13+1 using ASCA by Ueda et al. (2001) who detected a narrow absorption feature at $7.01 \mathrm{keV}$ which they interpreted as resonant scattering of the $\mathrm{K} \alpha$ line from $\mathrm{H}$-like Fe. XMM-Newton observations revealed an even more complex picture for GX 13+1 with narrow absorption features identified with the $\mathrm{K} \alpha$ and $\mathrm{K} \beta$ transitions of $\mathrm{He}$ - and $\mathrm{H}$-like iron (Fe XXV and Fe XXVI) and H-like calcium (Ca XX) K $\alpha$ detected (Sidoli et al. 2002). There is also evidence for the presence of a deep Fe XXV absorption edge at $8.83 \mathrm{keV}$ and a broad emission feature at around $6.4 \mathrm{keV}$. XMM-Newton observations of the eclipsing and dipping LMXRB MXB 1658-298 revealed narrow absorption features identified with $\mathrm{O}$ VIII $\mathrm{K} \alpha, \mathrm{K} \beta$, and $\mathrm{K} \gamma$, Ne X K $\alpha, \mathrm{Fe}$ XXV K $\alpha$, and Fe XXVI K $\alpha$ together with a broad Fe emission feature at $6.47 \mathrm{keV}$ (Sidoli et al. 2001). Another LMXRB dip source, X 1624-490, also displays K $\alpha$ absorption lines identified with Fe XXV and Fe XXVI as well as fainter absorption features tentatively identified with Ni XXVII K $\alpha$ and Fe XXVI K $\beta$. A broad emission feature at $6.58 \mathrm{keV}$ is also evident (Parmar et al. 2002). The properties of the absorption features in the $3 \mathrm{LBXRBs}$ show no obvious dependence on orbital phase, except during a dip from X 1624-490 where there is evidence for the presence of additional cooler material. Here we report the discovery of narrow X-ray absorption features from highly ionized Fe in the XMM-Newton spectrum of X 1254-690. As we discuss below, the presence of X-ray absorption features is probably related to the viewing angle of the system.

\section{Observations}

The XMM-Newton Observatory includes three $1500 \mathrm{~cm}^{2}$ $\mathrm{X}$-ray telescopes each with an EPIC at the focus. Two of the EPIC imaging spectrometers use MOS CCDs (Turner et al. 2001) and one uses a PN CCD. The region of sky containing X 1254-690 was observed by XMM-Newton between 2001 January 22 15:49 UT to 20:03 UT and again between 2002 February 07 17:32 UT to February 08 01:08 UT. In order to minimize the effects of pile-up the PN was operated in Timing mode during both observations. In this mode only one central CCD is read out with a time resolution of $0.03 \mathrm{~ms}$. This provides a one dimensional ( $4.4^{\prime}$ wide) image of the source with the second dimension being replaced by timing information. The faster CCD readout results in a much higher count rate capability of $1500 \mathrm{~s}^{-1}$ before charge pile-up become a serious problem. One MOS camera was operated in Small Window mode and the other was operated in Timing mode. As the PN effective area is a factor 5 higher than the MOS one at around $7 \mathrm{keV}$ where the absorption features are discovered, we concentrate here on the analysis of PN data. The PN camera was operated with thin filters during both observations.

Raw data products were extracted from the public XMM-Newton archive and processed using version 5.4.1 of the Science Analysis Software (SAS). Only single and double X-ray events corresponding to patterns $0-4$ were selected.
Known hot, or flickering, pixels and electronic noise were rejected using the SAS. Both observations contain high background intervals due to solar activity. These intervals were excluded for the spectral analysis.

Source events were extracted from a column 70" wide centered on X 1254-690. Background events were obtained from a column of the same width, but centered $100^{\prime \prime}$ from X 1254-690. The background events will be contaminated by a small number of source events due to the extent of the point spread function. However, background subtraction is not critical for such a bright source. The mean count rates during both observations of 175 and $185 \mathrm{~s}^{-1}$ are well below the value $\left(1500 \mathrm{~s}^{-1}\right)$ where source pile-up becomes a problem in PN Timing mode.

\section{Results}

\subsection{X-ray lightcurves}

The $0.5-10$ keV X 1254-690 background subtracted lightcurves obtained from the PN during the two observations are shown in Fig. 1 with a binning of $20 \mathrm{~s}$. The upper panels show the $\mathrm{PN}$ hardness ratios (counts in the energy range 0.5-10 keV divided by those between $0.5-3 \mathrm{keV}$ ) also with a binning of $20 \mathrm{~s}$. The source intensity was less during the 2001 observation with a mean count rate of $185 \mathrm{~s}^{-1}$ compared to $175 \mathrm{~s}^{-1}$ during the 2002 February observation. During the 2001 January observation a deep dip occurs between $19.0 \mathrm{hrs}$ and $19.6 \mathrm{hrs}$ on 22 January 2001 with irregular intensity variability and an associated increase in hardness ratio. During the dip the count rate drops as low as $70 \mathrm{~s}^{-1}$ when plotted at $20 \mathrm{~s}$ time resolution. Figure 2 shows an expanded view of the dip with a time resolution of $4 \mathrm{~s}$. At times, the dipping in almost total in the $0.5-10 \mathrm{keV}$ energy range when plotted with this time resolution. In the 2002 observation, the $0.5-10 \mathrm{keV}$ source count rate varies between $\sim 170-200 \mathrm{~s}^{-1}$ with flare-like variability, but there is no evidence for any dipping activity.

\subsection{X-ray spectra}

In order to minimize the effect of any background variations on the extracted spectra, intervals where the $>10 \mathrm{keV}$ PN count rate (one CCD) was $<1.4 \mathrm{~s}^{-1}$ were selected. These selected intervals are indicated by a thick horizontal line on top of Fig. 1. For the 2001 spectrum the dip was excluded. This resulted in exposures of $9.7 \mathrm{ks}$ and $12.5 \mathrm{ks}$ for the 2001 and 2002 observations, respectively. The spectra were rebinned to oversample the full width half-maximum of the energy resolution by a factor 3 and to have additionally a minimum of 20 counts per bin to allow use of the $\chi^{2}$ statistic. In order to account for systematic effects a $2 \%$ uncertainty was added quadratically to each spectral bin. The photo-electric absorption cross sections of Morrison \& McCammon (1983) are used throughout. All spectral uncertainties are given at $90 \%$ confidence. An initial examination of the 2001 and 2002 spectra showed that they are remarkably similar and in what follows we discuss the results of the (longer) 2002 February observation and indicate where the 2001 January results differ significantly. 

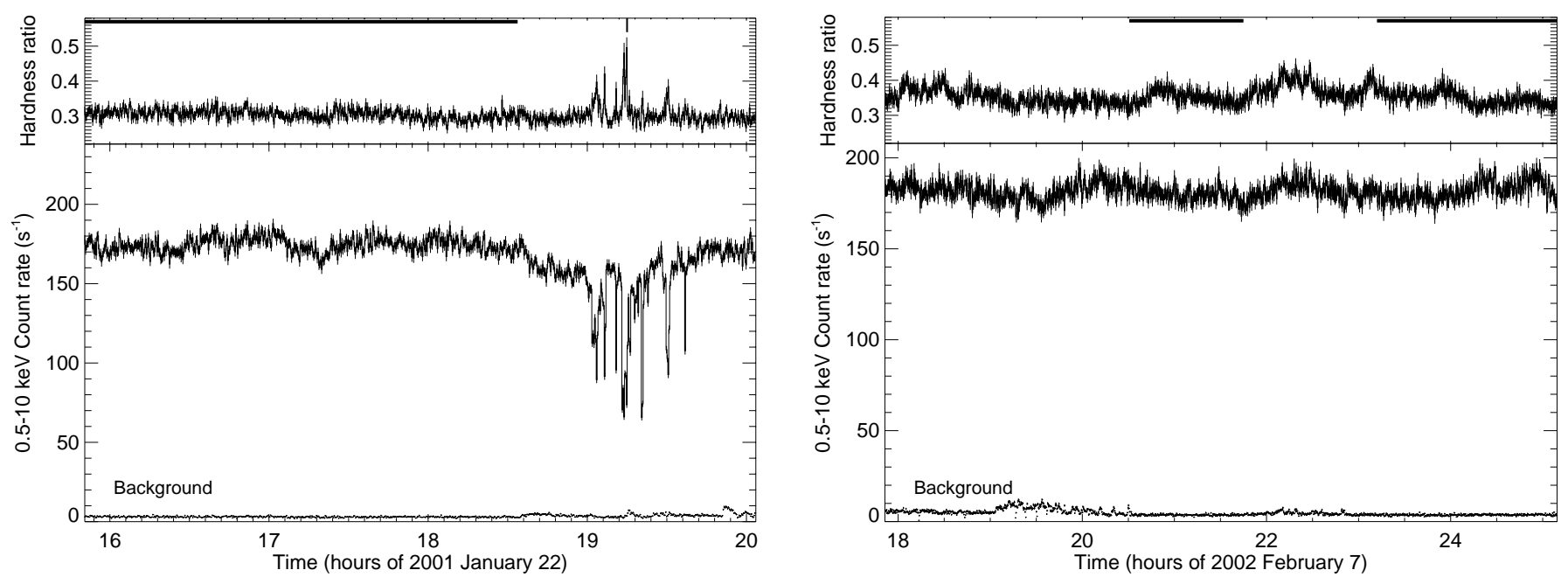

Fig. 1. The EPIC PN 0.5-10 keV background subtracted lightcurves of X 1254-690 with a binning of $20 \mathrm{~s}$ (lower panels) showing a deep dip during the 2001 observation and the lack of dipping activity during the 2002 observation. The upper panels show the hardness ratios (counts between 3-10 keV divided by those between $0.5-3 \mathrm{keV}$ ) also with a binning of $20 \mathrm{~s}$. The $0.5-10 \mathrm{keV}$ background counts taken from regions adjacent to the source are also shown. The thick horizontal lines indicate the intervals included in the spectral analysis. The tick mark flags the estimated dip center time chosen as phase 0 .

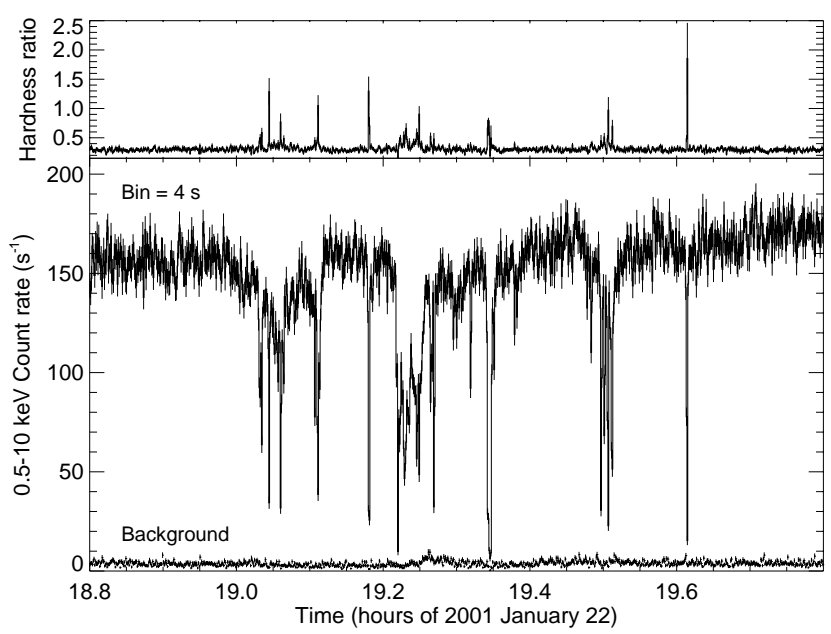

Fig. 2. A $0.5-10 \mathrm{keV}$ PN background subtracted lightcurve of the dip seen in the 2001 January observation plotted with a time resolution of $4 \mathrm{~s}$ showing the rapid intensity variability during the dip. The upper panel shows the hardness ratio (counts between $3-10 \mathrm{keV}$ divided by those between $0.5-3 \mathrm{keV}$ ).

The overall continuum observed in 2002 February was modeled using absorbed multicolor disk-blackbody and powerlaw components. In the multicolor disk-blackbody (Mitsuda et al. 1984; Makishima et al. 1986) $R_{\text {in }}$ is the innermost radius of the disk, $i$ the disk inclination angle and $k T_{\text {in }}$ the blackbody effective temperature at radius $R_{\text {in }}$. A power-law was chosen rather than the Comptonization model used by Iaria et al. (2001) or the cutoff power-law used by Smale et al. (2002) due to the more limited energy range of the EPIC instrument. This models the overall shape of the continuum moderately well and gives a $\chi^{2}$ of 392.1 for 234 degrees of freedom (d.o.f.). In contrast to the RXTE results of Smale et al. (2002), the disk-blackbody provides significantly better fits than a blackbody, and no cutoff to the power-law is needed at $\sim 6 \mathrm{keV}$. Examination of the residuals (middle panels of Fig. 3) reveals broad structure between 1-2 keV. This is probably the same structure noted by Iaria et al. (2001) using BeppoSAX and modeled as an absorption edge at $1.27 \mathrm{keV}$ and an optical depth, $\tau$, of 0.15 . In order to model this structure in the PN spectrum, an absorption edge at $1.20 \pm 0.04 \mathrm{keV}$ with $\tau=0.051 \pm 0.013$ was added to the model. This results in a $\chi^{2}$ of 346.9 for 232 d.o.f. However, significant structured residuals remain. These are much better accounted for if the edge is replaced by a Gaussian emission feature with an energy of $0.93_{-0.09}^{+0.05} \mathrm{keV}$, a width $(\sigma)$ of $175 \pm 65 \mathrm{eV}$ and an equivalent width, $E W$, of $29 \pm 13 \mathrm{eV}$ to give a $\chi^{2}$ of 312.7 for 231 d.o.f. The F-statistic value of 19.6 for the addition of the line feature indicates that the probability of such a decrease occurring by chance is $3 \times 10^{-11}$. The narrow features at $1.8 \mathrm{keV}$ and $2.2 \mathrm{keV}$ visible in Fig. 3 may be due to an incorrect modeling of the instrumental $\mathrm{Si}$ and Au edges.

Examination of the remaining fit residuals in both spectra shows deep negative residuals at $\sim 7 \mathrm{keV}$ (Fig. 4). These were modeled by a Gaussian absorption line with an energy of $6.96 \pm$ $0.04 \mathrm{keV}$, a width $(\sigma)$ of $<95 \mathrm{eV}$ and an equivalent width $(E W)$ of $-21_{-5}^{+8} \mathrm{eV}$. This results in a $\chi^{2}$ of 264.8 for 228 d.o.f. The F-statistic value of 13.7 indicates that the probability of such a decrease occurring by chance is $3 \times 10^{-8}$. It is probable that a second narrow $(\sigma<80 \mathrm{eV})$ absorption feature is present at an energy of $8.16 \pm 0.06 \mathrm{keV}$ with an $E W$ of $-16_{-8}^{+9} \mathrm{eV}$. Including such a feature in the fit results in a $\chi^{2}$ of 244.6 for 225 d.o.f. The F-statistic value of 6.2 indicates that the probability of such a decrease occurring by chance is only $5 \times 10^{-4}$. The best-fit energy is close to that of Fe XXVI K $\beta$ at $8.26 \mathrm{keV}$, a feature seen in the ASCA spectrum of GRS $1915+105$ by Kotani et al. (2000) and probably in the XMM-Newton spectrum of X 1624-490 by Parmar et al. (2002). We caution that this is a spectral region 

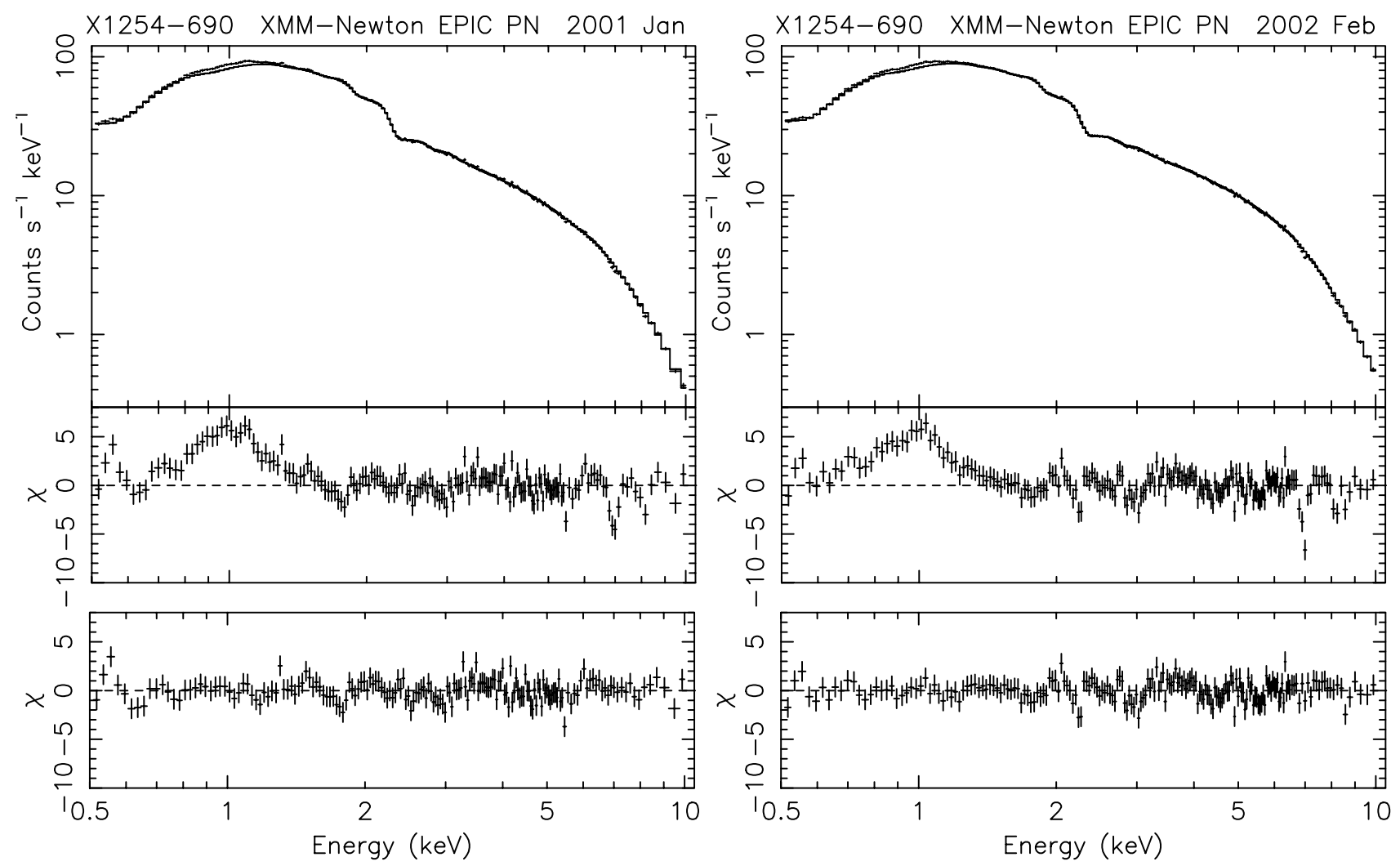

Fig. 3. The upper panels show the PN spectra of the X 1254-690 persistent emission and the best-fit disk-blackbody and power-law continuum models. The residuals (middle panels) reveal the presence of a broad emission feature centered at $\sim 0.93 \mathrm{keV}$, together with narrow absorption features at $6.96 \mathrm{keV}$ and $8.16 \mathrm{keV}$. The lower panels show the residuals when these features are included in the spectral model.

where the EPIC calibration is still relatively uncertain, and so we regard the presence, and proposed identification, of the feature as probable, but not confirmed. The best-fit continuum and line model parameters for both observation are given in Table 1. The $90 \%$ confidence upper-limit to a narrow Fe XXV absorption line at $6.70 \mathrm{keV}$ in the 2002 February observation is $-6 \mathrm{eV}$.

\subsection{Orbital dependence of the spectral features}

In order to investigate whether the properties of the absorption features depend on orbital phase, the persistent emission interval of the 2001 observation was divided into three parts of $\sim 3220 \mathrm{~s}$. To estimate the phases covered by these intervals, we use a period of $3.88 \mathrm{~h}$ and the time $19.25 \mathrm{~h}$ of 2001 January 22, corresponding to the apparent dip center (see Fig. 1), as an arbitrary reference for phase 0 . The same continuum model as used for the total persistent spectrum was fit to the 3 PN spectra. In addition, the Fe XXVI K $\alpha$ absorption feature is present. The Fe XXVI K $\beta$ absorption feature is not significantly detected. The properties of the Fe XXVI K $\alpha$ absorption features in the 3 segments are given in Table 2 . They are all consistent with those obtained from the total persistent spectrum. Thus, there does not appear to be any obvious dependence of the Fe XXVI K $\alpha$ absorption feature properties on orbital phase during the persistent emission.

\section{Discussion}

We have succesfully modeled the $0.5-10 \mathrm{keV}$ continuum of X 1254-690 during non-dip intervals using a combination of disk-blackbody and power-law models together with a broad emission feature at $\sim 0.95 \mathrm{keV}$ and narrow absorption features at $7.0 \mathrm{keV}$ and $8.2 \mathrm{keV}$. The nature of the emission feature is uncertain. The same structure was probably modeled as an absorption edge by Iaria et al. (2001) from BeppoSAX data, but the form of this feature clearly depends on the underlying continuum model chosen. For example, if a blackbody is substituted for the disk-blackbody in the 2002 February fits, then the emission feature has an energy of $0.98 \pm 0.02 \mathrm{keV}$ and a width of $<100 \mathrm{eV}$. Thus while the data clearly deviate from the chosen underlying continuum in this region, the exact form of the deviation is uncertain. We note, however, that the properties of the narrow absorption features are only very weakly dependent on the overall continuum model chosen.

Narrow X-ray absorption lines were first detected from the superluminal jet sources GRO J1655-40 (Ueda et al. 1998; Yamaoka et al. 2001) and GRS 1915+105 (Kotani et al. 2000; Lee et al. 2002). ASCA observations of GRO J1655-40 revealed the presence of absorption features due to FeXXV and FeXXVI which did not show any obvious dependence of their $E W \mathrm{~s}$ on orbital phase. ASCA observations of GRS 1915+105 revealed, in addition, absorption features due to CaXX, NiXXVII and Ni XXVIII. A recent Chandra HETGS observation of this source revealed absorption edges 
Table 1. Best-fits to the 0.5-10 keV XMM-Newton PN persistent emission spectra of X 1254-690 using a disk-blackbody model with an inner temperature $K T_{\text {in }}$ and normalization $k$ given by $\left(R_{\mathrm{in}} / d\right)^{2}$ where $R_{\mathrm{in}}$ in the inner radius in $\mathrm{km}$ and $d$ the distance in units of $10 \mathrm{kpc}$ and a power-law with a photon index, $\alpha$ together with an emission and two absorption lines.

\begin{tabular}{llcc}
\hline \hline Component & Parameter & 2001 January & 2002 February \\
\hline & $N_{\mathrm{H}}\left(10^{21}\right.$ atom cm $\left.{ }^{-2}\right)$ & $3.2 \pm 0.1$ & $3.1 \pm 0.1$ \\
& $L\left(\mathrm{erg} \mathrm{s}^{-1}\right.$, at $\left.10 \mathrm{kpc}\right)$ & $1.110^{37}$ & $1.010^{37}$ \\
& $\chi^{2} / \mathrm{d} .0 . f$. & $264.6 / 225$ & $244.6 / 225$ \\
Disk-blackbody & $k T_{\text {in }}(\mathrm{keV})$ & $1.97_{-0.07}^{+0.04}$ & $2.31 \pm 0.03$ \\
& $k$ & $1.41 \pm 0.10$ & $1.06 \pm 0.04$ \\
Power-law & $\alpha$ & $2.20 \pm 0.09$ & $2.34 \pm 0.09$ \\
& $1 \mathrm{keV}$ normalization & $0.106^{3} \pm 0.006$ & $0.099 \pm 0.005$ \\
Emission line & $E_{\text {line }}(\mathrm{keV})$ & $0.96_{-0.06}^{+0.04}$ & $0.93_{-0.09}^{+0.05}$ \\
& $\sigma(\mathrm{eV})$ & $175_{-50}^{+75}$ & $175 \pm 65$ \\
Fe XXVI K $\alpha$ abs & $E W(\mathrm{eV})$ & $32^{2} \pm 15$ & $29 \pm 13$ \\
feature & $E_{\text {line }}(\mathrm{keV})$ & $6.95 \pm 0.03$ & $6.96 \pm 0.04$ \\
& $\sigma(\mathrm{eV})$ & $<120$ & $<95$ \\
Fe XXVI K $\beta$ abs & $E W(\mathrm{eV})$ & $-27_{-8}^{+11}$ & $-21_{-5}^{+8}$ \\
feature & $E_{\text {line }}(\mathrm{keV})$ & $8.20_{-0.10}^{+0.05}$ & $8.16 \pm 0.06$ \\
& $\sigma(\mathrm{eV})$ & $<170$ & $<80$ \\
& $E W(\mathrm{eV})$ & $-17_{ \pm} \pm 9$ & $-16 \pm 9$ \\
\hline
\end{tabular}

Table 2. Properties of the Fe XXVI K $\alpha$ absorption feature in three segments of the persistent emission during the 2001 observation. The time reference used for phase 0 is $19.25 \mathrm{~h}$ of 2001 January 22 and the period is $3.88 \mathrm{~h}$.

\begin{tabular}{cccc}
\hline \hline & Segment 1 & Segment 2 & Segment 3 \\
\hline Phase range & $0.12-0.35$ & $0.35-0.59$ & $0.59-0.82$ \\
Fe XXVI K $\alpha$ abs feature & & & \\
$E_{\text {line }}(\mathrm{keV})$ & $6.93 \pm 0.09$ & $6.95_{-0.06}^{+0.03}$ & $6.97_{-0.05}^{+0.04}$ \\
$\sigma(\mathrm{eV})$ & $<185$ & $<140$ & $<126$ \\
$E W(\mathrm{eV})$ & $-22_{-15}^{+16}$ & $-21 \pm 11$ & $-31_{-12}^{+14}$ \\
\hline
\end{tabular}

of $\mathrm{Fe}, \mathrm{Si}, \mathrm{Mg}$, and $\mathrm{S}$ as well as resonant absorption features from Fe XXV and Fe XXVI and possibly Ca XX (Lee et al. 2002). Until recently, it was possible that these absorption features were peculiar to superluminal jet sources and related in some way to the jet formation mechanism. With the discovery of narrow absorption features from the LBXRBs GX 13+1 (Ueda et al. 2001), MXB 1658-298 (Sidoli et al. 2001), X 1624-490 (Parmar et al. 2002) and now X 1254-690, this appears not to be the case, and as proposed by Kotani et al. (2000) ionized absorption features may be common characteristics of disk accreting systems. However, it is interesting to note that 3 of the above 4 LMXRBs are dipping sources. These are systems that are viewed from directions close to the plane of their accretion disks with $i \sim 60-80^{\circ}$ (Frank et al. 1987). Furthermore, GRO J1655-40 has been observed to undergo deep absorption dips (Kuulkers et al. 1998) consistent with observing the source at an inclination angle, $i$, of $60^{\circ}-75^{\circ}$ (e.g., Frank et al. 1987). An inclination of $69.5 \pm 0.3^{\circ}$ is independently attributed to GRO J1655-40 from optical observations (Orosz \& Bailyn 1997). An inclination of $\sim 70^{\circ}$ is attributed to GRS $1915+105$ assuming that the jets are perpendicular to the accretion disk (Mirabel \& Rodriguez 1994). This suggests that inclination angle is important in determining the strength of these absorption features which implies that the absorbing material is distributed in a cylindrical, rather than a spherical geometry, around the compact object. The azimuthal symmetry is implied by the lack of any orbital dependence of these features.

The upper-limit to a narrow Fe XXV absorption feature from X 1254-690 of $-6 \mathrm{eV}$ and the Fe XXVI $E W$ of $-21_{-5}^{+8} \mathrm{eV}$ implies a ratio of $\mathrm{H}$-like to He-like line $E W \mathrm{~s}$ of $\gtrsim 2.7$. Comparison of this ratio with those from the above LBXRBs shows that only X 1624-490 has a comparable value of $2.2_{-2.0}^{+0.6}$ (Parmar et al. 2002), whilst for MXB 1658-298 the $E W$ ratio is $1.3_{-0.9}^{+0.4}$ (Sidoli et al. 2001) and for GX $13+1$ it is $1.6_{-1.0}^{+0.3}$, $1.5_{-0.9}^{+0.4}$ and $1.2 \pm 0.4$ during three different observations (Sidoli et al. 2002). This suggests that the material responsible for the absorption features in X 1254-690 is more strongly ionized than in MXB 1658-298 and GX 13+1. This difference is unlikely to be caused by observing the central source through hotter material located closer to the disk since MXB 1658-298 

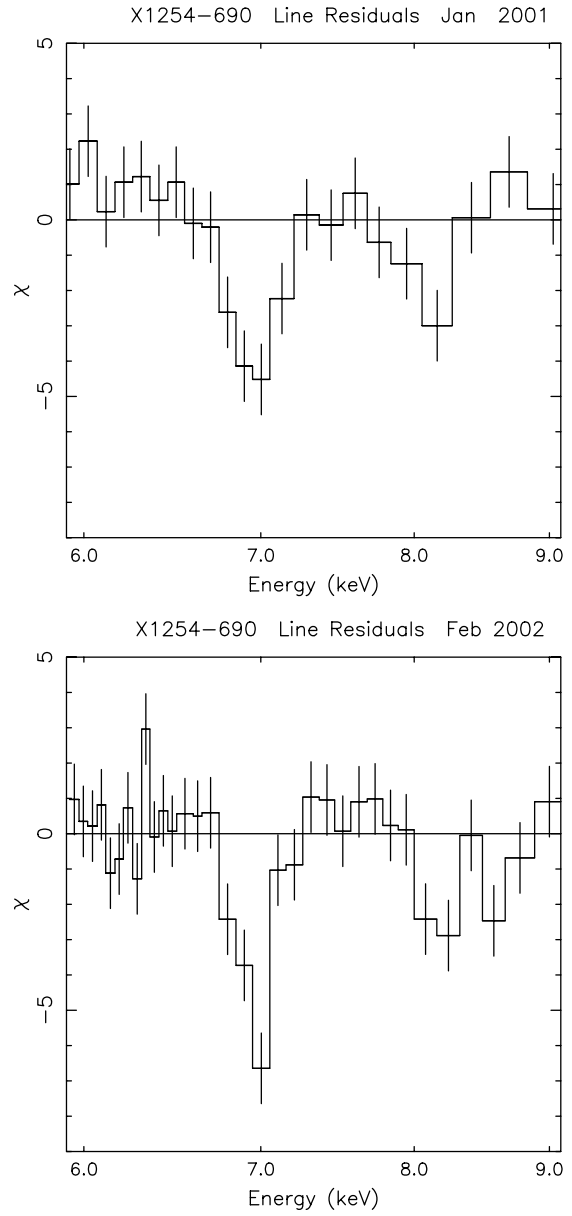

Fig. 4. Residuals in the 6.0-9.0 keV energy range when the best-fit models given in Table 1 are fit to the PN spectra of the persistent emission and the normalisations of the narrow absorption features at $6.96 \mathrm{keV}$ and $8.16 \mathrm{keV}$ are set to zero.

which shows eclipses and dips and has $i \sim 72-90^{\circ}$ (Cominsky $\&$ Wood 1989) has a $E W$ ratio of $1.3_{-0.9}^{+0.4}$. (However, the lines from MXB 1658-298 have the highest overall $E W$ ). It is also unlikely to be related to the total source luminosity since GX 13+1 with 1-10 keV luminosity of $7 \times 10^{37} \mathrm{erg} \mathrm{s}^{-1}$ and an $E W$ ratio of $\sim 1.4$ is around a factor 7 more luminous than $\mathrm{X} 1254-690$. One possibility is that this difference is related to the overall size of the Roche lobe around the compact object into which the accretion disk must fit. Systems with shorter orbital periods are therefore expected to have smaller accretion disks. The orbital period of $3.9 \mathrm{hr}$ for X 1254-690 is signicantly less than for MXB 1658-298 (7.1 hr), X 1624-490 (21 hr) and GX 13+1 (593 hr). Since the photo-ionization parameter, $\xi=L / n_{\mathrm{e}} r^{2}$, (see e.g., Kallman \& McCray 1982) where $L$ is the luminosity of the ionizing source, $n_{\mathrm{e}}$ is the electron density and $r$ is the distance to the ionizing source, depends on $r^{-2}$, the obscuring material in smaller systems may be expected to be more photo-ionized than in larger systems. This may well explain the high ionization level of the absorbing material in X 1254-690. On the basis of the properties of the Fe emission feature seen from XB 1916-053, Parmar et al. (2002) predicted that this $0.53 \mathrm{hr}$ dip source should exhibit absorption features. If the high ionization state of the absorbing material in X 1254-690 is due to its closeness to the central object, then the same should be true in the case of XB 1916-053.

Acknowledgements. Based on observations obtained with XMM-Newton, an ESA science mission with instruments and contributions directly funded by ESA member states and the USA (NASA). L. Boirin acknowledges an ESA Fellowship.

\section{References}

Cominsky, L. R., \& Wood, K. S. 1989, ApJ, 337, 485

Courvoisier, T. J.-L., Parmar, A. N., Peacock, A., \& Pakull, M. 1986, ApJ, 309, 265

Frank, J., King, A. R., \& Lasota, J.-P. 1987, A\&A, 178, 137

Iaria, R., Di Salvo, T., Burderi, L., \& Robba, N. R. 2001, ApJ, 548, 883

Jansen, F., Lumb, D., Altieri, B., et al. 2001, A\&A, 365, L1

Kallman, T., \& McCray, R. 1982, ApJS, 50, 263

Kotani, T., Ebisawa, K., Dotani, T., et al. 2000, ApJ, 539, 413

Kuulkers, E., Wijnands, R., Belloni, T., et al. 1998, ApJ, 494, 753

Lee, J. C., Reynolds, C. S., Remillard, R., et al. 2002, ApJ, 567, 1102

Makishima, K., Maejima, Y., Mitsuda, K., et al. 1986, ApJ, 308, 635

Mirabel, I. F., \& Rodriguez, L. F. 1994, Nature, 371, 46

Mitsuda, K., Inoue, H., Koyama, K., et al. 1984, PASJ, 36, 741

Morrison, D., \& McCammon, D. 1983, ApJ, 270, 119

Motch, C., Pedersen, H., Beuermann, K., Pakull, W. W., \& Courvoisier, T. J.-L. 1987, ApJ, 313, 792

Orosz, J. A., \& Bailyn, C. D. 1997, ApJ, 477, 876

Parmar, A. N., Oosterbroek T., Boirin, L., \& Lumb, D. 2002, A\&A, 386,910

Sidoli, L., Oosterbroek, T., Parmar, A. N., et al. 2001, A\&A, 379, 540

Sidoli, L., Parmar, A. N., Oosterbroek, T., \& Lumb, D. 2002, A\&A, 385, 940

Smale, A. P., Church, M. J., \& Bałucińska-Church, M. 2002, ApJ, 581, 1286

Smale, A. P., \& Wachter, S. 1999, ApJ, 527, 341

Strüder, L., Briel, U., Dennerl, K., et al. 2001, A\&A, 365, L18

Turner, M. J. L., Abbey, A., Arnaud, M., et al. 2001, A\&A, 365, L27

Ueda, Y., Inoue, H., Tanaka, Y., et al. 1998, ApJ, 492, 782

Ueda, Y., Asai, K., Yamaoka, K., Dotani, T., \& Inoue, H. 2001, ApJ, 556, L87

Uno, S., Mitsuda K., Aoki, T., \& Makino, F. 1997, PASJ, 49, 353

White, N. E., \& Swank, J. H. 1982, ApJ, 253, L61

Yamaoka, K., Ueda, Y., Inoue, H., et al. 2001, PASJ, 53, 179 